Cahiers
de a Recherche
sur les Droits Fondamentaux

\section{Cahiers de la recherche sur les droits} fondamentaux

15 | 2017

Le corps humain saisi par le droit : entre liberté et propriété

\title{
Naissances blanches - le deuil périnatal entre propriété du corps de l'enfant et liberté du sujet
}

"Sham Births"- Perinatal Bereavement between Ownership of the Body of the Child and the Freedom of the Subject

Corinne Chaput-Le Bars, Thierry Chartrin et Gilles Raoul-Cormeil

\section{OpenEdition}

\section{Journals}

Édition électronique

URL : https://journals.openedition.org/crdf/560

DOI : $10.4000 /$ crdf.560

ISSN : 2264-1246

\section{Éditeur}

Presses universitaires de Caen

\section{Édition imprimée}

Date de publication : 1 novembre 2017

Pagination : 115-122

ISBN : 978-2-84133-858-0

ISSN : $1634-8842$

\section{Référence électronique}

Corinne Chaput-Le Bars, Thierry Chartrin et Gilles Raoul-Cormeil, « Naissances blanches - le deuil périnatal entre propriété du corps de l'enfant et liberté du sujet », Cahiers de la recherche sur les droits fondamentaux [En ligne], 15 | 2017, mis en ligne le 01 octobre 2019, consulté le 15 novembre 2022. URL : http://journals.openedition.org/crdf/560 ; DOI : https://doi.org/10.4000/crdf.560 


\title{
Naissances blanches - le deuil périnatal entre propriété du corps de l'enfant et liberté du sujet
}

\author{
Corinne CHAPUT-LE BARS \\ Directrice du département Recherche, développement des formations supérieures \\ et partenariats universitaires à I'Institut régional du travail social (IRTS) Normandie-Caen \\ Thierry CHARTRIN \\ Gilles RAOUL-CORMEIL \\ Maître de conférences (HDR) en droit privé à l'université de Caen Normandie \\ Institut Demolombe (EA 967)
}

Responsable du service Recherche, prospective et développement à l'Association régionale pour I'Institut de formation en travail social (ARIFTS) à Angers

Introduction: variations sur le blanc

I. L'acte d'enfant sans vie ou le droit entre gris clair et gris foncé

A. Les conditions de l'acte d'enfant sans vie

1. Le critère biologique

2. Le critère juridique

B. Les effets de l'acte d'enfant sans vie

1. Exposé des effets

2. Critique des effets

II. De l'ondoiement aux rites hospitaliers, des rituels inventés pour adoucir la couleur du deuil

Introduction: variations sur le blanc ${ }^{1}$

Cet article à trois voix est né de l'émergence toute récente d'une notion qui reste à explorer et à approfondir pour en faire un concept. Les naissances blanches ${ }^{2}-$ titre donné
«D'Étienne, nous n'avons rien connu. Nous n'avons pas eu le temps de l'aimer», dit Jules à Valentine. Et Valentine de penser que seul un homme peut prononcer ces mots.

Extrait du film Éternité, réalisé par Trần Anh Hùng, 2016.

à notre conférence à trois voix en préambule au colloque «Le corps humain saisi par le droit: entre liberté et propriété»-, c'est par cette expression que l'une d'entre nous a désigné les naissances d'enfants sans vie ou morts dans les premières heures ou les premiers jours de leur vie, avant

1. Par Corinne Chaput-Le Bars.

2. Voir C. Chaput-Le Bars, «Introduction : naître ou ne pas naître», in Histoires de naissances, naissance d'histoires, C. Chaput-Le Bars (dir.), Paris, L'Harmattan, 2016, p. 27-28. 
même que les rituels ordinaires d'accueil et d'annonce dans la communauté humaine n'aient eu le temps de se réaliser «dans les formes». Elle lui avait été inspirée par un récit bouleversant envoyé comme contribution à un ouvrage collectif par une femme qui racontait la mort de sa dernière fille mort-née en laissant, sans en avoir la conscience même, une page blanche seulement numérotée, en fin de texte, comme pour mieux dire la solitude d'une mère face à cette naissance sans bébé, à une mise au monde sans monde à construire pour elle ${ }^{3}$.

Il y a quelques années, un numéro thématique de la revue Le sociographe, intitulé Petites discriminations ordinaires, avait permis à Pape Dieng, l'un de ses auteurs ${ }^{4}$, de proposer une variation sur le mot «noir». Aujourd'hui, le mot «blanc» se prête à la même grille de lecture et force est de constater, malgré un préjugé plus favorable, qu'il n'est guère moins péjoratif.

Très négatif en effet est le blanc quand il est synonyme d'imperfection, d'insuccès, voire de violence. En poésie, les "vers blancs» sont ceux qui ne riment pas. «Être connu comme le loup blanc» n'est pas gage de qualité, loin s'en faut. "Être cousu de fil blanc» manque singulièrement de discrétion. Quant à "saigner à blanc», c'est conduire autrui à l'épuisement. Et que dire de "faire chou blanc», expression berrichonne qui signifiait à l'origine "faire coup blanc» au jeu de quilles? Nul ne l'ignore: il s'agit ici de subir un échec cinglant.

Moins virulentes mais tout aussi pénibles sont les expressions de la blancheur quand elles désignent le vide, le manque, l'absence. Le «bulletin blanc» placé dans l'urne est un bulletin qui ne mentionne le nom d'aucun candidat. Le « rapport blanc» est un document non signé. Le «chèque en blanc» ne comporte aucune indication de montant. «Donner un blanc-seing» à quelqu'un consistait autrefois à lui remettre un document vierge et signé. La "nuit blanche» nous conduit dans les affres du manque de sommeil. Et l'expression "noir sur blanc» insiste sur l'aspect formel de l'écriture quand la «page blanche» de l'écrivain décrit la panne d'inspiration.

Il est une autre signification de la blancheur dans la langue française, en apparence plus anodine, qu'il nous faut pourtant observer de plus près. Faire quelque chose sans conséquence, expression d'abord employée dans le langage militaire, a donné naissance à la «balle à blanc», qui remplace le meurtrier projectile par une poudre blanche inoffensive. Un « examen blanc» est une préparation avant l'épreuve officielle, qui ne permet pas l'obtention du diplôme quand bien même la performance eût été remarquable. Un «mariage blanc» est un mariage non consommé, un mariage «pour de faux» comme pourraient le formuler les enfants, un «mariage qui fait semblant $»^{5}$.
En revanche, le blanc désigne bien entendu la pureté et l'innocence mais il est dit «cassé» quand il dépeint une couleur blanche légèrement teintée de jaune ou de gris. N'est-ce pas de tout cela qu'il s'agit ici? Des naissances au coloris blanc cassé?

Une naissance blanche est en effet tout à la fois une naissance ratée, une naissance imparfaite, une naissance douloureuse, une naissance "sans conséquence», une naissance "pour de faux», mais aussi une béance, celle d'un projet parental non abouti, celle d'un «enfant éternel», pour reprendre l'expression de Philippe Forest ${ }^{6}$. Quoi de plus innocent, en effet, qu'un enfant qui n'a pas eu le temps de vivre? Qui de plus idéalisé que le nouveau-né parti dès qu'il est advenu, nécessairement paré de toutes les vertus? "Là où l'enfant qui vit a toutes les chances de décevoir un jour, quoi qu'il fasse, l'enfant disparu n'a pas le temps de décevoir $» 7$.

Comment le droit a-t-il, au fil des ans, pris en compte cette souffrance humaine et sociale, si longtemps tue, déniée, aujourd'hui mieux entendue, mieux admise? Ce petit corps sans vie a-t-il désormais une existence juridique? Reconnaît-on qu'il appartient à une famille? Quelles libertés sont-elles concédées aux parents d'inscrire cet enfant dans leur histoire mais aussi à l'enfant qui vient après afin qu'il puisse conquérir une identité propre?

Les rituels de l'arrivée au monde, autrefois bâclés quand ils n'étaient pas absents, ont-ils eux aussi évolué dans les pratiques soignantes, sociales, religieuses et juridiques?

\section{L'acte d'enfant sans vie ou le droit entre gris clair et gris foncé ${ }^{8}$}

Selon l'article $1^{\mathrm{er}}$ de la Déclaration des droits de l'homme et du citoyen du 26 août 1789 dont la valeur normative est constitutionnelle, «Les hommes naissent et demeurent libres et égaux en droits. Les distinctions sociales ne peuvent être fondées que sur l'utilité commune». L'égalité entre les personnes physiques est portée si haut et depuis si longtemps par notre société contemporaine hexagonale qu'il peut sembler inutile de dissocier la vie biologique et l'existence juridique. Inutile en effet dès lors que les êtres humains sont en vie: ils jouissent de la même personnalité juridique. L'intérêt de la distinction entre la vie biologique et l'existence juridique se retranche aux frontières de la vie, à son orée puis à son crépuscule.

D'un côté, la vie biologique commence au jour de la conception de l'embryon mais l'être humain en gestation n'a pas d'existence juridique distincte de sa mère. Il est pars

3. J. Sanchez, "Cinq naissances», in Histoires de naissances..., p. 139-16o.

4. P. Dieng, «Noir, c'est noir... Témoignage d'un éducateur», Le sociographe, $\mathrm{n}^{\circ}$ 31, 2010, Petites discriminations ordinaires, $\mathrm{p}$. 25-30.

5. Sur la variété des mariages blancs saisie par le droit, voir G. Raoul-Cormeil, «Dissimulation et mariages blancs», in Droit et dissimulation (Actes du colloque tenu à Caen, 20-21 octobre 2011), A. Cerf-Hollender (dir.), Bruxelles, Bruylant (Penser le droit), 2013, p. 41-61.

6. P. Forest, L'enfant éternel, Paris, Gallimard, 1997.

7. C. Chaput-Le Bars, Naissance d'outre-tombe ou le complexe de Chateaubriand, Paris, L'Harmattan, 2014, p. 47.

8. Par Gilles Raoul-Cormeil. 
viscerum matris jusqu'au jour de l'accouchement; cette partie des entrailles de la femme enceinte est un élément du corps humain de la mère. Comme tel, le fotus est ainsi protégé car il est uni à sa mère par le cordon ombilical ${ }^{9}$. En revanche, au moment même où il est séparé de sa mère, le fotus acquiert la personnalité juridique pourvu qu'il soit né vivant et viable. Au jour de la naissance, l'octroi de la personnalité juridique bénéficie même d'un effet rétroactif depuis le jour de la conception chaque fois que le nouveauné avait eu un intérêt patrimonial à recevoir un bien ou une universalité de droits dans l'intervalle où il était en gestation: infans conceptus pro nato habetur quoties de commodis ejus agitur. Tel est le cas de l'enfant simplement conçu, dont le père est mort avant sa naissance. Né vivant et viable, l'enfant dont la filiation paternelle est établie peut être considéré comme existant au jour de l'ouverture de la succession de son père ${ }^{10}$.

À l'autre bout de la vie, la mort biologique marque l'extinction de la personnalité juridique. La dépouille mortelle de l'être humain sans vie est une chose sacrée à l'égard de laquelle la loi impose le respect. Le cadavre n'est pas créancier de ce droit au respect, faute d'être sujet de droit, mais l'ensemble des sujets de droit en vie doivent le traiter avec décence et dignité ${ }^{11}$. Le patrimoine du défunt n'a plus de maître. Ainsi, au jour même du décès, la succession du défunt est ouverte et ses héritiers présomptifs sont admis de plein droit à entrer en possession des biens de la succession dans le respect des droits des indivisaires jusqu'au jour du partage successoral. La loi accepte de prolonger la personnalité juridique dans l'incertitude biologique de la mort dans un délai de dix à vingt ans; tel est le but de la présomption légale d'absence qui, grâce à un jugement du juge des tutelles, permet de désigner l'administrateur des biens du présumé absent.

Le temps qui sépare la naissance et le décès importe peu dès lors qu'il est certain que l'être humain est né vivant et viable. L'être humain dont la vie autonome a duré un jour, un an, une décennie ou un siècle jouira d'un acte de naissance et d'un acte de décès ${ }^{12}$. Mais si l'être humain n'acquiert jamais les conditions biologiques d'une vie autonome, alors il n'a guère besoin d'accéder à la scène juridique ${ }^{13}$. L'être humain né sans vie ne revêtira pas le masque de la personnalité juridique; il ne sera pas un sujet de droit ${ }^{14}$. Chacune de ces deux hypothèses est traitée séparément dans les deux alinéas de l'article 79-1 du Code civil, introduit par la loi no 93-22 du 8 janvier 1993.

L'acte d'enfant sans vie est porté sur les registres du décès. Comment expliquer qu'un être humain ait un accès direct aux registres de décès sans passer préalablement par les registres de naissance? Ne faut-il pas avoir vécu pour mourir? Pas en droit, il ne sert à rien à l'enfant sans vie de revêtir le masque de la personnalité juridique: s'il n'est pas né vivant et viable, il n'est pas juridiquement une personne. Dépourvu de personnalité juridique, il n'aura ni patrimoine ni droits à céder ou à transmettre. Il n'aura jamais contracté; il n'aura jamais vécu sur la scène juridique. Le droit recherche la simplicité et l'efficacité. Pour autant, en figurant sur l'acte de décès, il oblige le maire, qui cumule la qualité d'officier de l'état civil et de gardien de l'ordre public sanitaire, ainsi responsable du sort des cadavres sur le territoire de la commune, à organiser les funérailles que lui réserve sa famille. C'est à ce titre que l'acte d'enfant sans vie est une très ancienne pratique administrative instituée par un décret du 4 juillet 1806 , en vigueur jusqu'à ce que la loi du 8 janvier 1993 ne l'abroge. Précisons toutefois que, de 1806 à 1919, l'officier de l'état civil auquel il fallait présenter le nouveau-né dans le but d'éviter la fraude à la conscription nationale ${ }^{15}$, déclarait que l'enfant lui avait été présenté sans vie, ce qui ne préjugeait pas de savoir s'il avait ou non vécu. Il était alors possible à celui qui prétendait qu'il avait vécu, de saisir le tribunal de grande instance d'une action en déclaration judiciaire de naissance

9. On a pu défendre que l'embryon entre dans la catégorie de «la personne par destination». Ainsi, en attendant que l'embryon n'accède à la vie autonome et ne reçoive la personnalité juridique, il est protégé par celle de sa mère. Sur cette qualification, voir X. Labbée, La condition juridique du corps humain avant la naissance et après la mort, Lille, Presses universitaires de Lille, 1990, p. 251; J.-P. Baud, «Le corps, personne par destination", in Droit des personnes et de la famille: mélanges à la mémoire de Danièle Huet-Weiller. Liber amicorum, Strasbourg - Paris, Presses universitaires de Strasbourg - LGDJ, 1994, p. 13-17; P. Murat, «Décès périnatal et individualisation juridique de l'être humain», Revue de droit sanitaire et social, 1995, p. 451-474, spéc. p. 468.

10. Art. 725 du Code civil. Il est de l'intérêt du nouveau-né d'acquérir un capital d'assurance décès ou d'assurance sur la vie si son existence d'enfant à naître a été prise en considération dans le contrat et a commencé au jour du décès du souscripteur; voir Cass., $1^{\text {re }}$ civ., 10 décembre $1985, \mathrm{n}^{\circ} 84-14.328$, Bulletin civil I, no 339: Recueil Dalloz, 1987, jurispr., p. 449, note G. Paire; Gazette du Palais, 1986, II, sommaires, p. 323, obs. A. Piedelièvre; Defrénois, 1986, art. 33722, p. 668, note G. Paire; Revue trimestrielle de droit civil, 1987, p. 309, obs. J. Mestre. Voir aussi Cass., $1^{\mathrm{re}}$ civ., 28 janvier 2009, nº 07-14.272: Recueil Dalloz, 2009, p. 1927, note G. Raoul-Cormeil.

11. Art. 16-1-1 du Code civil. Sur lequel, voir G. Loiseau, «Mortuorum corpus: une loi pour le respect (commentaire de la loi $\mathrm{n}^{\circ}$ 20o8-1350 du 19 décembre 2008)», Recueil Dalloz, 2009, p. 236-237.

12. Art. 79-1, al. $1^{\text {er }}$ du Code civil: «Lorsqu'un enfant est décédé avant que sa naissance ait été déclarée à l'état civil, l'officier de l'état civil établit un acte de naissance et un acte de décès sur production d'un certificat médical indiquant que l'enfant est né vivant et viable et précisant les jours et heures de sa naissance et de son décès".

13. En ce sens, voir L. Mayaux, Droit civil. Les personnes: cours et parcours, Paris, Ellipses, 1997, $\mathrm{n}^{\circ} 25$ in fine: «[...] s'il apparaît critiquable, ce pragmatisme témoigne d'un attachement à une conception fonctionnelle de la personnalité. Celle-ci serait un outil conféré à l'homme pour faciliter sa vie sociale; elle s'inscrirait dans la durée. L'enfant non viable qui a été privé du temps de vivre n'en aura pas besoin ».

14. Art. 79-1, al. 2 du Code civil: "À défaut du certificat médical prévu à l'alinéa précédent, l'officier de l'état civil établit un acte d'enfant sans vie. Cet acte est inscrit à sa date sur les registres de décès et il énonce les jour, heure et lieu de l'accouchement, les prénoms et noms, dates et lieux de naissance, professions et domiciles des père et mère et, s'il y a lieu, ceux du déclarant. L'acte dressé ne préjuge pas de savoir si l'enfant a vécu ou non; tout intéressé pourra saisir le tribunal de grande instance à l'effet de statuer sur la question».

15. Voir L. Leveneur, «La différenciation des sexes en droit privé contemporain», in Le sexe, la sexualité et le droit (Actes du XVII ${ }^{e}$ colloque national de la Confédération des juristes catholiques de France), Paris, P. Téqui, 2002, p. 45 sq., spéc. p. 77. L'un des buts avoués de cette formalité était d'éviter que «des garçons, soldats à venir, ne fussent déclarés comme filles». 
pour qu'il acquiert la personnalité juridique et, avec elle, un patrimoine à transmettre à ses héritiers ${ }^{16}$. À compter de la loi du 20 novembre 1919 qui a supprimé l'obligation de présenter le nouveau-né à l'officier de l'état civil ${ }^{17}$, ce fut au médecin d'indiquer au père ou à la personne qui déclarait la naissance que l'enfant était sans vie ${ }^{18}$.

Qu'on se place sous l'empire du décret de 1806 ou sous celui de la loi de 1993, la question est la même: l'acte d'enfant sans vie est-il un acte de l'état civil comme les autres? On a pu en douter et soutenir qu'il était imparfait ${ }^{19}$. À l'inverse, on a pu défendre la «délicatesse $»^{20}$ du droit qui accompagne la douleur de la famille endeuillée. Dans cette voie, un auteur a su convaincre que l'enfant sans vie pourrait bénéficier d'un état civil complet sans cependant jouir de la personnalité juridique ${ }^{21}$. Près de trente ans après sa forte démonstration, le droit positif a évolué sans aller jusqu'à consacrer sa thèse doctrinale. L'enfant sans vie n'a ni personnalité juridique, ni état civil complet. Alors qui se soucie de l'enfant sans vie? De la famille de la parturiente endeuillée ou de la société, qui garde en mémoire le souvenir de l'existence de l'enfant sans vie? La formalité administrative est-elle mise en œuvre pour le passé, le présent ou l'avenir?

À la lumière des textes et de la jurisprudence, les conditions (A) et les effets de l'acte d'enfant sans vie (B) sortent difficilement de l'ambiguïté. Entre gris clair et gris foncé, le positionnement du droit doit être expliqué avant d'être critiqué.

\section{A. Les conditions de l'acte d'enfant sans vie}

Les conditions de l'acte d'enfant sans vie ont évolué opportunément pour des raisons tenant aux sources du droit. Au fond, les progrès de la science et de l'imagerie médicale n'expliquent pas à eux seuls l'abaissement du critère biologique autorisant l'officier de l'état civil à rédiger l'acte d'enfant sans vie. Il existe des seuils de résistance d'ordre culturel qui justifient l'introduction d'un critère formel ou juridique.

\section{Le critère biologique}

Pendant de longues décennies, la pratique administrative de l'acte d'enfant sans vie était réservée à deux types de fotus. Le fotus mort-né, tout d'abord, désigne le nouveauné qui est mort au cours de la vie intra-utérine; il est expulsé par la mère dans les conditions d'un accouchement. La césarienne n'est pas une condition. Le médecin qui accouche la parturiente constate la mort du foetus. La situation est distincte du fœetus qui est né vivant mais non viable. C'est le cas ensuite du fotus condamné. La loi n’ayant pas défini la viabilité, celle-ci a été subordonnée à un double critère. Positivement, le fotus doit être parvenu à une «maturité suffisante ${ }^{22}$. Cette gestation minimale a longtemps été évaluée à cent-quatre-vingts jours ${ }^{23}$, parce qu'elle correspondait au premier délai de la présomption légale de durée de la grossesse ${ }^{24}$. Négativement, le fotus non viable est celui qui n'a pas la constitution organique pour vivre ou survivre ${ }^{25}$. Sa constitution anormale le prive de la capacité naturelle de vivre ${ }^{26}$. Sur cette base, l'enfant viable est celui dont le sexe est déterminable et dont les traits corporels sont ceux d'un être humain. Autrement dit, n'est pas viable l'embryon qui n'est qu'amas de cellules. Les progrès de la science ont rendu ces critères obsolètes...

La loi du 8 janvier 1993 n'a toutefois pas pris le parti de définir la viabilité. Des médecins se sont émus de l'absence de critère biologique et ont souhaité la consécration des recommandations de l'Organisation mondiale de la santé (OMS). Les limites de la viabilité avaient été abaissées à vingt-deux semaines d'aménorrhée (soit vingt semaines

16. Voir P. Murat, «Décès périnatal et individualisation juridique de l'être humain », p. 460.

17. Art. 55 du Code civil (rédaction de la loi du 20 novembre 1919): «Les déclarations de naissance seront faites dans les trois jours de l'accouchement, à l'officier de l'état civil du lieu». Sur la suppression de la vérification du sexe du nouveau-né, voir A. Colin, H. Capitant, Cours élémentaire de droit civil français, $8^{\mathrm{e}}$ éd., Paris, Dalloz, 1934, t. I, n 402, p. 412; M. Planiol, G. Ripert, Traité élémentaire de droit civil, $12^{\mathrm{e}}$ éd., Paris, LGDJ, 1937, t. I, n ${ }^{\circ}$ 492. En réaction à la croisade entreprise par le $D^{r}$ Loir entre 1845 et 1865 contre la présentation du nouveau-né à l'officier de l'état civil, une circulaire du ministre de l'Intérieur du 9 avril 1870 a préconisé la constatation de la naissance par des médecins délégués par l'administration municipale.

18. En ce sens, voir A. Colin, H. Capitant, Cours élémentaire de droit civil français, $\mathrm{n}^{\circ} 403$, p. 413 : «Le décret du 4 juillet 1806 décide que, lorsque l'enfant meurt avant la déclaration de naissance, l'officier de l'état civil dresse un acte sur le registre des décès, mais sans qu'il en résulte aucun préjugé sur la question de savoir si l'enfant a eu vie ou non. Dans cet acte, l'officier constate qu'il a reçu la déclaration que l'enfant n'était pas vivant ».

19. En ce sens, voir X. Labbée, La condition juridique du corps humain..., p. 104: «Si la déclaration est obligatoire, l'inscription sur le registre de l'état civil n'emporte aucune conséquence».

20. G. Cornu, Droit civil. Les personnes, $13^{\mathrm{e}}$ éd., Paris, Montchrestien (Domat droit privé), 2007, no 10 in fine, p. 22. À rapprocher de G. Mémeteau, «Vie biologique et personnalité juridique (Qui se souvient des hommes?) ", in La personne humaine, sujet de droit (4 ${ }^{\text {es }}$ journées René Savatier, 25-26 mars 1993), Paris, PUF (Publications de la Faculté de droit de Poitiers), 1994, p. 21-56.

21. En ce sens, voir X. Labbée, La condition juridique du corps humain..., p. 104; cité par P. Murat, «Décès périnatal et individualisation juridique de l'être humain", p. 468.

22. P. Malaurie, Cour de droit civil. Les personnes, les incapacités, $1^{\mathrm{re}}$ éd., Paris, Cujas, 1992, $\mathrm{n}^{\circ} 4$; rééd.: $8^{\mathrm{e}}$ éd., Paris, Defrénois, 2016 , $\mathrm{n}^{\circ} 6$, p. 13 .

23. Cass., civ., 7 août 1874, Recueil Dalloz, 1875, I, p. 5: «L'enfant n'est réputé viable qu'après un minimum de 180 jours, ou six mois de gestation». Voir aussi G. Mémeteau, "La situation juridique de l'enfant conçu. De la rigueur classique à l'exaltation baroque», Revue trimestrielle de droit civil, 1990, p. 611-624.

24. Art. 311 du Code civil. La référence au délai de cent-quatre-vingts jours a été longtemps énoncée par l’Instruction générale relative à l'état civil $(\$ 461)$, citée par P. Murat, «Décès périnatal et individualisation juridique de l'être humain», p. 456.

25. A. Batteur, Droit des personnes, des familles et des majeurs protégés, 8 éd., Issy-les-Moulineaux, LGDJ, $2015, \mathrm{n}^{\circ} 24$.

26. C. Philippe, "La viabilité de l'enfant nouveau-né», Recueil Dalloz, 1996, chron., p. 29. Voir aussi L. Sebag, La condition juridique des personnes physiques et des personnes morales avant leur naissance, thèse de doctorat, université de Paris, 1938, p. 230; P. Salvage, «La viabilité de l'enfant nouveau-né», Revue trimestrielle de droit civil, 1976, p. 725-749; X. Labbée, La condition juridique du corps humain..., p. 49. 
de grossesse) ou un fotus ayant atteint le poids de 500 grammes. Ce double critère a été repris par la circulaire du 3 mars $1993^{27}$ puis par celle du 30 novembre $2001^{28}$.

Mais en France les circulaires administratives ne lient pas les juges de l'ordre judiciaire ${ }^{29}$; elles ne portent qu'une interprétation que le juge peut tenir pour inexistante si elle s'ajoute à la loi. C'est ainsi que dans trois arrêts de cassation rendus pour violation de l'article 79-1, alinéa 2 du Code civil, la Cour de cassation a jugé que ce texte «ne subordonne l'établissement de l'acte d'enfant sans vie ni au poids du fœtus, ni à la durée de la grossesse ${ }^{30}$. En l'espèce, chacun des trois fotus, dont le poids variait de 155 à 400 grammes, méritait d'être porté sur les actes de l'état civil. La Cour de cassation obligeait les pouvoirs publics à définir le seuil de la viabilité et à choisir entre un nouveau critère biologique et un critère formel.

\section{Le critère juridique}

En toute discrétion, les pouvoirs publics ont pris deux décrets le 20 août $2008^{31}$ et deux arrêtés ministériels le même jour ${ }^{32}$ en prenant soin de communiquer au grand public que les conditions de rédaction d'un acte d'enfant sans vie ne remettaient pas en cause le régime de l'interruption volontaire de grossesse. L'acte d'enfant sans vie ne doit pas être interprété comme conférant, au fond, la personnalité juridique à l'embryon, précisait le garde des Sceaux dans un communiqué de presse.

Les nouveaux textes ont été rédigés conformément à la nouvelle jurisprudence de la Cour de cassation. Aucun seuil biologique n'a été fixé pour subordonner la rédaction d'un acte d'enfant sans vie. Le seuil de l'OMS aurait pu être consacré par la loi ou celle-ci aurait pu autoriser le gouvernement à le définir par un décret d'application. Mais les pouvoirs publics ont préféré le silence de la loi plutôt que de créer un nouveau débat parlementaire sur les différentes facettes du statut juridique de l'embryon humain. Le gouvernement a pris appui sur la lettre de l'article $79-1$, alinéa $2^{\text {nd }}$ du Code civil qui subordonne la rédaction de l'acte d'enfant sans vie à la production d'un certificat médical établissant que l'enfant n'est pas né vivant et viable.
Le texte aurait pu définir la naissance, la vie et la viabilité. D'abord, la naissance est le fait de séparer l'embryon du sein de sa mère; la naissance suppose un acte d'expulsion. Ensuite, la vie est un autre fait biologique qui s'oppose à la mort qui n'est définie par le Code de la santé publique que dans le contexte particulier du prélèvement posthume d'organe. Enfin, la viabilité est le troisième fait biologique défini comme l'aptitude à vivre mais la prise en charge médicale ou chirurgicale des prématurés et des fotus mal formés conduit à analyser ce critère de manière libérale. C’est pourquoi le gouvernement a préféré ne pas définir ces trois critères et confier au médecin le soin de dire si l'enfant a été expulsé mort-né ou s'il est né vivant et non viable. La difficulté demeure: "Le tout reste alors de distinguer accouchement... et expulsion d'un foetus ${ }^{23}$.

En réalité, seuls deux cas ont été réservés par le décret pour interdire au médecin de rédiger le certificat médical d'accouchement. Il s'agit d'abord de l'interruption spontanée précoce de grossesse: la fausse couche précoce ne permet pas de rédiger d'acte d'accouchement, en l'absence d'un corps à forme humaine. Il s'agit ensuite de l'interruption volontaire de grossesse. L'acte d'enfant sans vie a pour but de faire le deuil d'une grossesse désirée; il n'est pas l'instrument d'une grossesse non désirée qui peut être librement refusée par la femme enceinte. L'interruption de grossesse pour un motif médical peut en revanche donner lieu à la rédaction d'un certificat médical d'accouchement, et cela même si cet acte médical est réalisé dans le délai de l'interruption volontaire de grossesse. L'interdiction faite au médecin de rédiger un acte d'accouchement dans ces deux cas ne convainc cependant pas tous les auteurs. Certains suggèrent ainsi l'alternative d'un seuil biologique dont l'effet d'exclusion aurait été plus neutre:

L'effet de seuil a l'avantage, il faut l'avouer, d'écraser les situations particulières: en l'occurrence, un délai de plus de douze semaines d'aménorrhée pour autoriser l'établissement de l'acte d'enfant sans vie aurait permis d'écarter ensemble, de manière innommée, les situations d'IVG et de fausse couche précoce, sans oublier celle de l'embryon in vitro détruit après conservation ${ }^{34}$.

27. Circulaire du 3 mars 1993 relative à l'état civil, à la famille et aux droits de l'enfant, Journal officiel de la République française, 24 mars 1993 , p. 4551.

28. Circulaire $\mathrm{n}^{\circ} 2001 / 576 \mathrm{du} 30$ novembre 2001 relative à l'enregistrement à l'état civil et à la prise en charge des corps des enfants décédés avant la déclaration de naissance, intégrée dans l'Instruction générale complémentaire relative à l'état civil du 29 mars 2002, Journal officiel de la République française, 28 avril 2002, p. 7719.

29. Cass., chambre commerciale, 23 octobre 1950 : Les grands arrêts de la jurisprudence civile, H. Capitant, F. Terré, Y. Lequette (dir.), $13^{\mathrm{e}}$ éd., Paris, Dalloz, 2015, t. I, n⿳0 13, p. 100-107, où les auteurs expliquent la divergence d'analyse avec le Conseil d'État.

30. Cass., $\mathrm{1}^{\mathrm{re}}$ civ., 6 février 2008, $\mathrm{n}^{\circ}$ 06-16.498: Droit de la famille, 2008, comm. 34, p. 21, note P. Murat; La semaine juridique, édition générale, no 11 , 2008, II, 10045, note G. Loiseau; Recueil Dalloz, 2008, p. 1442, obs. J.-C. Galloux, et p. 1862, note G. Roujou de Boubée et D. Vigneau; Revue juridique personnes et famille, avril 2008, p. 13, note F. Sauvage; Revue trimestrielle de droit civil, 2008, p. 268, obs. J. Hauser.

31. Décret $n^{\circ}$ 2008-798 du 20 août 2008 modifiant le décret $n^{\circ}$ 74-449 du 15 mai 1974 relatif au livret de famille, Journal officiel de la République française, 22 août 2008, p. 13144; décret no $2008-800$ du 20 août 2008 relatif à l'application du second alinéa de l'article 79-1 du Code civil, Journal officiel de la République française, 22 août 2008, p. 13145. Sur lesquels, voir G. Loiseau, "L'acte II d'enfant sans vie», Droit de la famille, 2008, comm. 135, p. 18; J. Massip, "Actes d'enfant sans vie: les deux décrets du 20 août 2008", Defrénois, 15 novembre 2008, art. 38850, p. 2148; J.-R. Binet, «Inscription à l'état civil des enfants nés sans vie», La semaine juridique, édition notariale et immobilière, $\mathrm{n}^{\circ} 37,2008,611$.

32. Arrêté du 20 août 2008 relatif au modèle de certificat médical d'accouchement en vue d'une demande d'établissement d'un acte d'enfant sans vie, Journal officiel de la République française, 22 août 2008, p. 13165; arrêté du 20 août 2008 modifiant l'arrêté du $1^{\text {er }}$ juin 2006 fixant le modèle du livret de famille, Journal officiel de la République française, 22 août 2008, p. 13145.

33. F. Terré, D. Laszlo-Fenouillet, Droit civil. Les personnes: personnalité, incapacité, protection, $8^{\mathrm{e}}$ éd., Paris, Dalloz (Précis Dalloz), 2012, nº $229, \mathrm{p} .223$.

34. G. Loiseau, «L'acte II d'enfant sans vie», p. 18. À rapprocher de M. Lamarche, «Actes d'enfant né sans vie, livret de famille et certificat médical: peut-on se satisfaire de la nouvelle réglementation?», Droit de la famille, 2008, alerte 67. 
La circulaire du 19 juin 2009 sur l'établissement d'un acte d'enfant sans vie semble s'être engagée dans cette voie puisqu'elle subordonne la délivrance d'un acte d'accouchement à une grossesse qui a durée quinze semaines d'aménorrhée, ce qui permet d'exclure sans le dire les interruptions volontaires ou accidentelles de grossesse du «premier trimestre ${ }^{35}$. Si le délai est satisfaisant, la méthode normative prête le flanc à la critique car la présente circulaire est dépourvue de force obligatoire à l'égard du juge judiciaire, comme l'a rappelé la Cour de cassation dans ses arrêts du 6 février 2008. La leçon de droit n'a pas été entendue par la Chancellerie!

Le nouveau régime des conditions de l'acte d'enfant sans vie suscite autant de critiques que ses effets.

\section{B. Les effets de l'acte d'enfant sans vie}

Les effets de l'acte d'enfant sans vie sont nombreux; ils sont même trop considérables pour être enfermés dans le cercle familial. Le deuil périnatal dépasse le cadre de la famille et intéresse la société tout entière, si bien que le législateur pourrait aller plus loin et offrir à l'enfant qui n'a pas vécu un état civil complet, à défaut d'une personnalité juridique qui n'aurait pas de sens. L'exposé des effets (1) précède donc la critique (2).

\section{Exposé des effets}

Les effets de l'acte d'enfant sans vie sont essentiellement familiaux. Le gouvernement a autorisé l'officier de l'état civil à remettre à la femme qui a perdu son enfant un livret de famille. Le livret de famille peut aussi être remis au couple de concubins qui, pour faire le deuil périnatal, demandent un acte d'enfant sans vie. Avant qu'il ne vienne à être réécrit par le décret du 20 août 2008, l'article 9 du décret du 15 mai 1974 était rédigé de telle sorte que l'instruction générale de l'état civil réservait la mention d'acte d'enfant sans vie aux couples qui disposaient déjà d'un livret de famille. Ainsi seuls les couples mariés et les couples non mariés mais ayant eu un premier enfant pouvaient faire mentionner sur leur livret de famille l'existence d'un enfant sans vie. Dorénavant, un livret de famille peut être remis à la seule femme qui a accouché ou au couple qu'elle forme avec la personne qui partage avec elle une communauté de vie, homme ou femme. C'est dire que l'enfant, même sans vie, fait famille ${ }^{36}$ ! Précisons que l'enfant sans vie ne sera désigné que par un prénom.

Le couple qui fait le deuil périnatal peut aussi réclamer le corps de l'enfant sans vie pour organiser ses obsèques ${ }^{37}$. Si les conditions de l'acte d'enfant sans vie sont réunies, le fotus expulsé ne peut pas être traité comme un déchet hospitalier: il ne doit pas être incinéré au four de l'hôpital avec les déchets humains issus des opérations chirurgicales mais il doit être traité, si telle est la volonté des porteurs du deuil périnatal, comme un cadavre humain ${ }^{38}$. Les obsèques conservent une nature familiale mais le caractère public de la sépulture du fotus humain confère un caractère social à l'acte d'enfant sans vie.

Enfin, la femme qui accouche d'un enfant sans vie peut prétendre au congé de maternité; le bénéfice du congé de paternité est ouvert à son mari ou à l'homme qui vit avec elle en concubinage et constitue le père putatif. Le bénéfice de ces congés ${ }^{39}$ révèle le rayonnement social du deuil périnatal; il serait donc réducteur de l'enfermer dans une dimension familiale. La femme qui doit subir une intervention médicale pour perdre son enfant sans vie est prise en charge par la société. Au congé de maternité, il faut ajouter d'autres droits financiers dont le bénéfice témoigne de l'alignement d'une grossesse interrompue pour un motif médical avec celle d'une grossesse qui parvient à son terme. La parturiente peut prétendre à une bonification de sa pension de retraite ${ }^{40}$, à une majoration de l'assurance-vieillesse en présence de trois enfants ${ }^{41}$ ou à la majoration du quotient familial en vue du calcul de l'impôt sur le revenu de l'année de l'accouchement ${ }^{42}$. L'accumulation de tous ces avantages d'ordre financier révèle l'épaisseur sociale que l'acte d'enfant sans vie a ainsi pu gagner au cours des dernières décennies.

\section{Critique des effets}

Le droit de l'acte d'enfant sans vie est en demi-teinte. L'impression d'un régime juridique inachevé est-elle justifiée ou critiquable?

Ainsi, d'un côté, l'enfant sans vie est dépourvu de la personnalité juridique et, comme tel, il ne bénéficie pas de tous les éléments identifiant l'enfant né vivant,

35. Circulaire interministérielle DGCL/DACS/DHOS/DGS/2009/182, 19 juin 2009, spéc. \$1.2.1.1: Droit de la famille, 2009, comm. 123, p. 19, note P. Murat; Revue trimestrielle de droit civil, 2010, p. 75, obs. J. Hauser.

36. À comparer avec I. Corpart, "Le foetus mort, enfant de personne», in De code en code. Mélanges en l'honneur du doyen Georges Wiederkehr, M. Puech (dir.), Paris, Dalloz, 2009, p. 133-145, spéc. p. 141, où l'auteure préconise la remise d'un certificat de maternité ou de paternité plutôt que la remise d'un livret de famille.

37. Art. R. 1112-75 sq. du Code de la santé publique. Voir aussi P. Murat, "Acte d'enfant sans vie: un mieux», Droit de la famille, 2002, comm. 48; I. Corpart, «Décès périnatal et qualification juridique du cadavre», La semaine juridique, édition générale, n 39, 2005, I, 171; D. Dutrieux, « De nouvelles règles en cas de décès en milieu hospitalier ", La semaine juridique, édition administrations et collectivités territoriales, $\mathrm{n}^{\circ} 37,2006,1200$.

38. Sur lequel, voir H. Popu, La dépouille mortelle, chose sacrée. À la redécouverte d'une catégorie juridique oubliée, thèse de doctorat, université de Lille, 2008.

39. Art. D. 331-4 et D. 613-10 du Code de la sécurité sociale.

40. Réponse ministérielle à la question écrite n² 2119, Journal officiel Sénat Questions, 11 septembre 2008, p. 1845.

41. Circulaire CNAV 2004/22 du 30 avril 2004; citée par I. Corpart, «Le fotus mort... », p. 141.

42. Courrier de la direction de la législation fiscale à l'association Petite Émilie, 19 septembre 2006. Voir aussi F. Sauvage, «L'acte d'enfant sans vie discrètement réglementé», Actualité juridique. Famille, octobre 2008, p. 392; Revue juridique personnes et famille, septembre 2008 , p. 15. 
viable, et titulaire d'un acte de naissance soumis au droit commun. L'enfant sans vie a un prénom, certes, mais il n'a ni nom de famille, ni filiation. La Russie qui a refusé à un couple l'établissement de la filiation d'un enfant mort-né s'est vue condamnée par la Cour européenne des droits de l'homme en $2005^{43}$. Douze ans plus tard, la législation française n'a toujours pas évolué, en dépit des critiques doctrinales portées de longue date par des auteurs partisans de conférer à l'enfant sans vie un état civil complet: sexe, prénom, nom, filiation, nationalité...

D'un autre côté, il faut rappeler que l'état civil est constitué de règles impératives qui accordent une place limitée à la volonté individuelle. Or, la constitution d'un acte d'enfant sans vie n'a rien d'obligatoire; elle est une faveur accordée par la loi pour aider les femmes qui accouchent d'un enfant mort-né ou né vivant et non viable à faire le deuil de leur grossesse interrompue. Cette faveur est essentielle à leur bien-être et à l'accueil de leur futur enfant. Mais le caractère thérapeutique et facultatif de cette législation marque une différence profonde avec l'état civil des sujets de droit dont l'existence intéresse la société tout entière pour le présent et les générations à venir ${ }^{44}$ (prévention de l'inceste, notamment). S'il emprunte les registres publics, l'acte d'enfant sans vie est un accompagnement solennel réalisé dans un but privé et familial: celui de faciliter le deuil d'un projet parental. Rien au fond n'oblige l'État et la société à se souvenir de l'existence des enfants sans vie; un tel souvenir n'a de sens que pour les personnes qui portent le deuil périnatal. C'est ce qui nous fait dire en somme que la législation française est subtile mais, au fond, parfaitement justifiée.

On pourrait aller plus loin dans la personnification de l'enfant sans vie mais cette évolution n'aurait-elle pas pour effet de remettre en cause la position de la Cour de cassation sur le refus de qualifier l'embryon de personne humaine au regard du Code pénal ${ }^{45}$ ? La subtilité du droit français est la marque d'un équilibre entre des positions contraires.

\section{De l'ondoiement aux rites hospitaliers, des rituels inventés pour adoucir la couleur du deuil ${ }^{46}$}

En nous intéressant à la question de la reconnaissance sociale de l'enfant sans vie, nous découvrons que, le 20 avril 2017 , cela a fait tout juste dix ans que l'Église catholique a enfin résolu d'abroger officiellement l'existence des «limbes», ce terrible non-lieu auquel étaient voués les enfants qui mouraient sans être baptisés ${ }^{47}$. Ce triste anniversaire nous rappelle alors la douloureuse histoire de ces parents endeuillés et les divers rites qu'ils ont dû élaborer au cours des siècles pour trouver de l'apaisement.

Face à la forte mortalité infantile, l'Église catholique a en effet mis beaucoup d'énergie à valoriser ces pertes inévitables : l'enfant qui mourait avant l'âge de 7 ans allait directement au paradis, sans condition ni purgatoire puisqu'il n'avait pas atteint l'âge du discernement du bien et du mal. Il devenait un ange de Dieu et pouvait alors intercéder auprès du Créateur pour les siens. Du fait de ce caractère providentiel, l'Église imposait un cérémonial spécifique dédié au décès de l'enfant où le noir laissait place au blanc, le rituel faisait référence à la fête plutôt qu'au chagrin, et les cloches sonnaient joyeusement plutôt que lugubrement. Mais encore fallait-il pour cela que l'enfant fût baptisé...

Pour ce faire, la religion catholique a inventé l'ondoiement. Il s'agit très exactement d'une bénédiction rapide, remplaçant le baptême « où seule l'ablution baptismale est faite, sans les rites et les prières habituels. L'ondoiement est donné à un enfant en danger de mort». Cette définition, tirée de la Conférence des évêques de France ${ }^{48}$, nous renseigne sur le caractère exceptionnel de la cérémonie car, pour qu'il y ait ondoiement, il doit y avoir un risque de mort imminente chez le nouveau-né. Il s'agit donc d'une cérémonie de l'urgence puisqu'elle concerne un rite non abouti pour un enfant non abouti (comprendre ici enfant qui ne va pas vivre ou dont on pense qu'il ne va pas vivre).

43. Cour EDH, 2 juin 2005, Znamenskaya c. Russie, $\mathrm{n}^{\circ}$ 77785/01: La semaine juridique, édition générale, $\mathrm{n}^{\circ} 30,2005, \mathrm{I}, 159$, obs. $\mathrm{F}$. Sudre, $\mathrm{n}^{\circ}$ 14; Revue trimestrielle de droit civil, 2005, p. 737, obs. J.-P. Marguénaud.

44. Pour aller plus loin, voir G. Raoul-Cormeil, «L'état civil, lieu de mémoire de l'existence sociale de la personne », in Le droit à la lumière de Bergson: mémoire et évolution, C. Puigelier, B. Saint-Sernin (dir.), Paris, Panthéon-Assas, 2013, p. 147-169.

45. Sur le refus d'admettre l'homicide par imprudence, infraction pénale contre la personne humaine, à l'égard d'un enfant à naître, voir D. Rebut, «La loi pénale est d'interprétation stricte!», Droit de la famille, 1999, chron. 20; G. Roujou de Boubée, B. de Lamy, «Contribution supplémentaire à l'étude de la protection pénale du fotus (à propos de l'arrêt de la chambre criminelle du 30 juin 1999)", Recueil Dalloz, 200o, chron., p. 181-183; J. Pradel, «La seconde mort de l'enfant conçu (à propos de l'arrêt d'Assemblée plénière du 29 juin 2001) ", Recueil Dalloz, 2001, p. 2907; Y. Mayaud, "Ultime complainte après l'arrêt d'Assemblée plénière de la Cour de cassation du 29 juin 2001 ", Recueil Dalloz, 2001, p. 2917; D. Vigneau, «Selon la Cour de cassation, l'homicide d'un enfant à naître, même viable, n'en est pas un! (à propos de Cass., ass. plén., 29 juin 2001)», Droit de la famille, 2001, chron. 21, p. 4-15. Cette interprétation a été jugée conforme à la Convention européenne des droits de l'homme: Cour EDH, 8 juillet 2004, Vo c. France, n 53924/o0. Sur cet arrêt, voir J. Pradel, «La CEDH, l'enfant conçu et le délit d'homicide involontaire: entre prudence et embarras", Recueil Dalloz, 2004, p. 2456. La position de la France n'est pas remise en cause. Aurait-on pu qualifier l'enfant à naître de personne humaine sans remettre en cause la liberté d'avorter? Toute la difficulté est de concilier une liberté et une protection ou, comme le proposent des auteurs, de distinguer selon que l'enfant à naître est l'objet ou non d'un projet parental. Sur cette proposition, voir G. Roujou de Boubée, "Grandeur et décadence de l'interprétation stricte (très brèves observations à propos de l'homicide par imprudence du fotus)", in Ruptures, mouvements et continuité du droit: autour de Michelle Gobert, Paris, Economica, 2004, p. 195-203; J.-Y. Chevallier, " "Naître ou n'être pas". La chambre criminelle et l'homicide du fotus", in Droit et actualité: études offertes à Jacques Béguin, Paris, Litec, 2005, p. 125-140; A. Decocq, «La main d'Isabelle», in Mélanges en l'honneur de Philippe Malaurie, Paris, Defrénois, 2005, p. 199-213.

46. Par Thierry Chartrin.

47. Je fais cette découverte grâce au texte de Béatrice Kammerer, «Les enfants non-baptisés ont-ils droit au paradis?», Slate, 26 avril 2016 , en ligne: www.slate.fr/story/117265/enfants-non-baptises-droit-paradis.

48. Site de la Conférence des évêques de france, glossaire: http://www.eglise.catholique.fr/glossaire/ondoiement. 
L'ondoiement puise sa raison d'être dans la dimension eschatologique de la religion judéo-chrétienne.

À notre époque, on ne peut imaginer à quel point la mort d'un enfant mort-né ou décédé avant qu'il soit baptisé emplissait les gens de frayeur et de chagrin. Si cela arrivait, il n'y avait pas de sacrement, ni d'office à l'église, pas de sépulture chrétienne et l'âme allait dans les limbes ${ }^{49}$.

Avec cet acte, si l'enfant décède, il aura la possibilité d'être inhumé chrétiennement. Si l'enfant survit, le sacrement traditionnel du baptême sera prononcé: il sera, en quelque sorte, baptisé deux fois! André Comte-Sponville souligne que la grande force des religions n'est pas tant de rassurer les croyants face à leur mort que la crainte de la damnation éternelle ${ }^{50}$.

En théorie, ce geste devait être effectué lorsque l'état de l'enfant laissait pressentir qu'il ne survivrait pas jusqu'à la cérémonie ordinaire. En pratique, le désarroi maternel était souvent tel que les ondoiements d'enfants mort-nés ont été nombreux entre le $\mathrm{XV}^{\mathrm{e}}$ et le XVII ${ }^{\mathrm{e}}$ siècle, souvent avec la complicité des accoucheuses. En parallèle, la pratique de l'ondoiement in utero, qui présentait l'avantage de ne pas avoir besoin d'apprécier la viabilité de l'enfant, s'est aussi développée. La sage-femme versait alors l'eau bénite sur la tête du fœtus avec la main ou au moyen d'une éponge tout en prononçant la formule du baptême, une technique perfectionnée aux XVIII ${ }^{\mathrm{e}}$ et XIX ${ }^{\mathrm{e}}$ siècles avec l'invention de seringues à baptiser, introduites par le vagin.

Sans ce précieux sésame, point de paradis, les âmes des petits enfants étant condamnées à errer dans les «limbes». Ce lieu neutre et intermédiaire, ni paradis ni enfer, fut à l'origine imaginé par les théologiens du $\mathrm{XII}^{\mathrm{e}}$ siècle pour tranquilliser les parents. Mort sans baptême et pourtant marqué du péché originel, l'enfant ne pouvait certes pas prétendre au paradis mais, sans avoir seulement vécu, comment pouvait-il mériter les flammes de l'enfer? Or, loin d'apaiser les parents, les limbes ont au contraire matérialisé leurs pires cauchemars et donné lieu pendant des siècles à bien des pratiques désespérées pour baptiser à tout prix.

Depuis lors, comme nous l'avons vu, le droit a commencé à prendre en compte cette souffrance sociale. Dans les hôpitaux normands, depuis les années 2010, les parents des enfants nés après quinze semaines d'aménorrhée, et qui ont «forme humaine», sont informés qu'ils peuvent organiser des funérailles et, même lorsque le corps de l'enfant est confié à l'établissement, celui-ci est désormais incinéré uniquement avec les cadavres d'autres enfants et ses cendres sont répandues sur un lieu dédié.

Enfin, les parents sont aujourd'hui autorisés à lui donner un ou des prénoms, qui figureront dans leur livret de famille et qui n'obligeront plus l'enfant qui vient après, cet enfant "d'outre-tombe», à porter administrativement la même identité que le disparu et à porter émotionnellement la charge qu'elle représente. 